TRANSACTIONS OF THE

AMERICAN MATHEMATICAL SOCIETY

Volume 348, Number 6, June 1996

\title{
BOUNDARY AND LENS RIGIDITY OF LORENTZIAN SURFACES
}

\author{
LARS ANDERSSON, MATTIAS DAHL, AND RALPH HOWARD
}

\begin{abstract}
Let $g$ be a Lorentzian metric on the plane $\mathbb{R}^{2}$ that agrees with the standard metric $g_{0}=-d x^{2}+d y^{2}$ outside a compact set and so that there are no conjugate points along any time-like geodesic of $\left(\mathbb{R}^{2}, g\right)$. Then $\left(\mathbb{R}^{2}, g\right)$ and $\left(\mathbb{R}^{2}, g_{0}\right)$ are isometric. Further, if $(M, g)$ and $\left(M^{*}, g^{*}\right)$ are two dimensional compact time oriented Lorentzian manifolds with space-like boundaries and so that all time-like geodesics of $(M, g)$ maximize the distances between their points and $(M, g)$ and $\left(M^{*}, g^{*}\right)$ are "boundary isometric", then there is a conformal diffeomorphism between $(M, g)$ and $\left(M^{*}, g^{*}\right)$ and they have the same areas. Similar results hold in higher dimensions under an extra assumption on the volumes of the manifolds.
\end{abstract}

\section{INTRODUCTION}

In this paper we consider uniqueness questions for Lorentzian manifolds where time-like geodesics have no conjugate points and the closely related problem of uniqueness for Lorentzian metrics on manifolds with boundaries in terms of the boundary distances. In the Riemannian setting the earliest uniqueness result for manifolds without conjugate points seems to be the theorem of E. Hopf [10] that a metric without conjugate points on a two dimensional torus is flat. (For extensions of this result and more references, cf. [8] and [3].) This result was used by Green and Gulliver [6] to show that a metric on the plane $\mathbb{R}^{2}$ with no conjugate points that agrees with the standard metric outside a compact set is flat. Our main result is that this result extends to Lorentzian metrics.

Theorem 1.1. Let $g_{0}=-d x^{2}+d y^{2}$ be the standard flat metric on $\mathbb{R}^{2}$ and $g$ any other Lorentzian metric on $\mathbb{R}^{2}$ that has no conjugate points along any time-like geodesic and which agrees with $g_{0}$ outside a compact set. Then $\left(\mathbb{R}^{2}, g\right)$ is isometric with $\left(\mathbb{R}^{2}, g_{0}\right)$.

In [5] Croke extended the Green and Gulliver result to Riemannian metrics on $\mathbb{R}^{n}$ without conjugate points and which agree with the standard metric outside a compact set. In doing so he showed that this result follows from the boundary rigidity for domains in Euclidean space, a problem which had already been considered by Gromov [7] and Michel [12]. In another paper Croke [4] gave a very

Received by the editors January 18, 1995.

1991 Mathematics Subject Classification. Primary 53C50.

Lars Andersson supported in part by the Swedish Natural Sciences Research Council (SNSRC), contract no. F-FU 4873-307. Mattias Dahl supported in part by the Wallenberg foundation. Ralph Howard supported in part by the SNSRC, contract no. R-RA 4873-306, the Swedish Academy of Sciences and the Crafoord foundation.

(C)1996 American Mathematical Society 
general boundary rigidity result for two dimensional Riemannian manifolds. In [4] Croke introduces the class of strongly geodesically minimizing manifolds, a class of compact manifolds that includes totally convex domains and which seems to be the natural class to consider when dealing with boundary rigidity questions. In order to define the analogous notion in the Lorentzian case we make the following definition.

Definition 1.2. A space-like slab is a time oriented Lorentzian manifold $(M, g)$ with non-empty boundary $\partial M$ which is space-like and so that every inextendible causal curve $c$ meets both the past boundary $N$ (the set of boundary points $p$ where the inward unit normal points to the future) and the future boundary $N_{+}$ (the set of boundary points where the inward normal points to the past), and that moreover the past and future boundaries are complete as Riemannian manifolds.

This implies that both $N$ and $N_{+}$are Cauchy hypersurfaces in $(M, g)$ and therefore $(M, g)$ is globally hyperbolic. (Our basic references for the concepts of Lorentzian geometry are $[1,9]$. For the rest of this paper we will denote by $N$ the past boundary and by $N_{+}$the future boundary of a space-like slab $(M, g)$.) The following class of space-times is the Lorentzian analogue of the strongly geodesically minimizing manifolds.

Definition 1.3. A space-like slab $(M, g)$ is strongly geodesically maximizing (or $\boldsymbol{S} \boldsymbol{G M}$ for short) iff every time-like geodesic $\gamma:[0, L] \rightarrow M$ of $(M, g)$ maximizes the distance between any two of its points in the strong sense that $\gamma(0)$ has no cut points in the closed interval $[0, L]$.

The following condition is not as natural as the condition SGM, but most of the spaces we are interested in satisfy it and it is very important for technical reasons in our proofs.

Definition 1.4. A space-like slab $(M, g)$ is free of cut points iff the past boundary $N$ has no cut point in $M$ along any geodesic normal to $N$.

We use the notation $p \ll q$ to mean that there is a time-like curve from $p$ to $q$. If $p \ll q$ then the Lorentzian distance $d(p, q)$ is the supremum of the lengths of all the time-like curves from $p$ to $q$ and $d(p, q)=0$ if $p \nless q$. Let $(M, g)$ and $\left(M^{*}, g^{*}\right)$ be two space-like slabs and let us assume there is an isometry $\varphi: \partial M \rightarrow \partial M^{*}$ (so that $\varphi$ is an isometry of the Riemannian manifolds $\left(\partial M,\left.g\right|_{\partial M}\right)$ and $\left.\left(\partial M^{*},\left.g^{*}\right|_{\partial M^{*}}\right)\right)$. Let $\eta$ be the future pointing unit vector field along $N$ and $\xi$ the future pointing unit vector field along $N_{+}$. Let $\eta^{*}$ and $\xi^{*}$ be the corresponding vector on $N^{*}$ and $N_{+}^{*}$. Let $H(N)$ be the bundle of all future pointing time-like unit vectors of $M$ based at a point of $N$ and $H\left(N_{+}\right), H\left(N^{*}\right)$ and $H\left(N_{+}^{*}\right)$ the corresponding bundles over $N_{+}$, $N^{*}$ and $N_{+}^{*}$. Then for any vector $V$ tangent to $N$ there is a unique number $\theta>0$ so that $\cosh (\theta) \eta+V \in H(N)$, that is so that $\cosh (\theta) \eta+V$ is a future pointing unit vector. Then $\varphi$ induces a map $\varphi_{\#}: H(N) \rightarrow H\left(N^{*}\right)$ by $\varphi_{\#}(\cosh (\theta) \eta+V)=$ $\cosh (\theta) \eta^{*}+\varphi_{*} V$. There is a similarly defined map $\varphi_{\#}: H\left(N_{+}\right) \rightarrow H\left(N_{+}^{*}\right)$.

Definition 1.5. Let $(M, g)$ and $\left(M^{*}, g^{*}\right)$ be space-like slabs.

1. A smooth map $\varphi: \partial M \rightarrow \partial M^{*}$ is a boundary isometry iff it is an isometry of the Riemannian manifolds $\left(\partial M,\left.g\right|_{\partial M}\right)$ and $\left(\partial M^{*},\left.g^{*}\right|_{\partial M^{*}}\right)$ and for all $p \in N$ and $q \in N_{+}$the equality $d^{*}(\varphi(p), \varphi(q))=d(p, q)$ holds.

2. A smooth map $\varphi: \partial M \rightarrow \partial M^{*}$ is a lens equivalence iff it is an isometry of $\partial M$ with $\partial M^{*}$ and if $v=\cosh (\theta) \eta+V \in H(N)$ and $\ell>0$ is so that $\exp (\ell v) \in$ 
$H\left(N_{+}\right)$, then for the geodesic $\gamma^{*}(t):=\exp ^{*}\left(t \varphi_{\#}(v)\right.$ it holds that $\gamma^{*}(\ell) \in N_{+}^{*}$ and $\varphi_{\#}\left(\gamma^{\prime}(\ell)\right)=\varphi\left(\left(\gamma^{*}\right)^{\prime}(\ell)\right)$. (That is if we identify $\partial M$ and $\partial M^{*}$ by $\varphi$, then for any inward pointing unit vector $v$ the corresponding time-like geodesics $\gamma$ and $\gamma^{*}$ with the initial velocity vector $v$ both have the same length, they both hit $N_{+}$at the same point $q$ and have the same velocity vector at $q$.)

3. The space-like slab $(M, g)$ is boundary rigid iff every space-like slab $\left(M^{*}, g^{*}\right)$ boundary isometric to $(M, g)$, is isometric to $(M, g)$. (Isometries are assumed to preserve the time orientations.)

4. The space-like slab $(M, g)$ is lens rigid iff every space-like slab $\left(M^{*}, g^{*}\right)$ lens equivalent to $(M, g)$ is isometric to $(M, g)$.

It will be shown in Section 2 that if $(M, g)$ is SGM and $\left(M^{*}, g^{*}\right)$ is boundary isometric to $(M, g)$, then $\left(M^{*}, g^{*}\right)$ is also SGM and $(M, g)$ and $\left(M^{*}, g^{*}\right)$ are lens equivalent. The only examples of boundary rigid space-like slabs known at present are

Theorem 1.6. Let $\left(N, d s^{2}\right)$ be a circle of length $L$ and let $2 w \leq L$. Then the Lorentzian cylinder $\left([0, w] \times N,-d t^{2}+d s^{2}\right)$ is both boundary and lens rigid.

In higher dimensions we show (Theorem 5.2) that if $(N, h)$ is a compact Riemannian manifold with injectivity radius $\geq w$ and $\left(M^{*}, g^{*}\right)$ is boundary isometric to $(M, g):=\left([0, w] \times N,-d t^{2}+h\right)$ and also $\operatorname{Vol}_{g}(M)=\operatorname{Vol}_{g^{*}}\left(M^{*}\right)$, then $(M, g)$ and $\left(M^{*}, g^{*}\right)$ are isometric.

For two dimensional space-like slabs the examples of Section 3.1 show the condition SGM, even along with the $(M, g)$ being free of cut points, is not enough to guarantee that $(M, g)$ is boundary rigid. However if $(M, g)$ is SGM, then at least its conformal type and area are determined by its boundary distances.

Theorem 1.7. Let $(M, g)$ be a SGM two dimensional space-like slab and let $\left(M^{*}, g^{*}\right)$ be boundary isometric to $(M, g)$. Then the boundary isometry extends to a conformal diffeomorphism between $(M, g)$ and $\left(M^{*}, g^{*}\right)$. If also $(M, g)$ is compact and free of cut points, then $(M, g)$ and $\left(M^{*}, g^{*}\right)$ have the same area.

Acknowledgments. Greg Galloway brought this circle of questions to our attention. Tilla Weinstein sent us a copy of [15] which suggested Proposition 6.1 to us. We have also benefitted from some conversations with Jan Boman on subjects related to this paper. Most this work was done while the third author (RH) was on sabbatical leave from the University of South Carolina at the Royal Institute of Technology.

\section{Preliminaries}

Let $(M, g)$ be a space-like slab and let $H(M)$ be the bundle of all future pointing time-like vectors tangent to $M$. For each $x \in M$, the fiber $H_{x}$ is the set of future pointing vectors satisfying $g(u, u)=-1$. Thus each fiber is isometric to the Riemannian hyperbolic space $\mathbf{H}^{n-1}$ with constant sectional curvature -1 . Let $\zeta^{t}$ be the geodesic flow on $H(M)$. That is for each $u \in H(M), \zeta^{t} u=\gamma^{\prime}(t)$ where $\gamma$ is the geodesic satisfying $\gamma^{\prime}(0)=u$. Let $H(N):=\bigcup\left\{H_{p}: p \in N\right\}$ be the restriction of $H(M)$ to the past boundary $N$. For $v \in H(N)$, let $\ell(v)$ be so that $\exp (v \ell(v)) \in N_{+}$ and let $\mathcal{H}:=\{(v, t) \in H(N) \times[0, \infty): t \leq \ell(v)\}$. Then because $N$ is a Cauchy hypersurface 
Proposition 2.1. The map $(v, t) \mapsto \zeta^{t} v$ From $\mathcal{H}$ to $H(M)$ is a diffeomorphism.

Let $\mathcal{M}:=\{(x, t) \in N \times[0, \infty): t \leq \ell(\eta(x))\}$. The following is also elementary.

Proposition 2.2. If $(M, g)$ is without cut points, then $(x, t) \mapsto \exp (t \eta(x))$ is a diffeomorphism of $\mathcal{M}$ with $M$.

The following two results show that it is possible to determine if $(M, g)$ is free of cut points or is SGM from the behavior of $d^{\partial}: N \times N_{+} \rightarrow(0, \infty)$.

Proposition 2.3. The space-like slab $(M, g)$ is free of cut points iff for all $q \in N_{+}$ there is a unique point $P(q)$ so that the function $d^{\partial}(\cdot, q)$ has a maximum at $P(q)$ and moreover the map $q \mapsto P(q)$ is a diffeomorphism of $N_{+}$with $N$. Thus if $(M, g)$ is free of cut points and $\left(M^{*}, g^{*}\right)$ is boundary isometric with $(M, g)$ then $\left(M^{*}, g^{*}\right)$ is also free of cut points.

Proposition 2.4. A space-like slab $(M, g)$ is $S G M$ iff for each $q \in N_{+}$the function $d^{\partial}(\cdot, q)$ is smooth on $N \cap I^{-}(q)$ and for each $p \in N$ the map $q \mapsto \nabla_{p} d^{\partial}(\cdot, q)$ is a diffeomorphism of $I^{+}(p) \cap N_{+}$with $H_{p}$. Thus if $(M, g)$ is $S G M$ and $\left(M^{*}, g^{*}\right)$ is boundary isometric to $(M, g)$ then $\left(M^{*}, g^{*}\right)$ is also $S G M$ and $(M, g)$ and $\left(M^{*}, g^{*}\right)$ are lens equivalent.

In the following proposition we will use the notation $\mathbb{R}^{n}[0, w]$ for the subset of $\mathbb{R}^{n}$ defined by $\mathbb{R}^{n}[0, w]:=\left\{x: 0 \leq x_{1} \leq w\right\}$. Recall that a time oriented Lorentzian manifold $(M, g)$ is future one connected iff for all $p, q \in M$ with $q \in I^{+}(p)$ and for all time-like curves $\gamma_{0}, \gamma_{1}:[0,1] \rightarrow M$ with $\gamma_{0}(0)=\gamma_{1}(0)=p$ and $\gamma_{0}(1)=\gamma_{1}(1)=q$ there is a homotopy of $\gamma_{0}$ to $\gamma_{1}$ through time-like curves keeping the endpoints fixed (cf. [1, Definition 9.28]).

Proposition 2.5. Let $g$ be a Lorentzian metric on $\mathbb{R}^{n}$ that agrees with the standard metric $g_{0}=-d x_{1}^{2}+d x_{2}^{2}+\cdots+d x_{n}^{2}$ outside a compact set $K \subset \mathbb{R}^{n}[0, w]$ and so that $\left(\mathbb{R}^{n}[0, w], g\right)$ is globally hyperbolic, future one connected, and the time-like geodesics of $\left(\mathbb{R}^{n}, g\right)$ have no conjugate points. Then $\left(\mathbb{R}^{n}[0, w], g\right)$ is both boundary isometric and lens equivalent to $\left(\mathbb{R}^{n}[0, w], g_{0}\right)$.

Remark 2.6. Part of the proof of Theorem 1.1 consists of showing that in two dimensions the assumptions on global hyperbolicity and future one connectedness are redundant (cf. Proposition 6.1), as any Lorentzian metric on the plane which agrees with the standard metric outside a compact set is globally hyperbolic and future one connected. In higher dimensions this is no longer true, cf. Remark 6.3; however it is still open if there is a counterexample that is free of time-like conjugate points.

Proof of 2.3. If $(M, g)$ is free of cut points, then each point $q \in N_{+}$is uniquely of the form $q=\exp (t \eta(p))$ for some $p \in N$ and $t>0$. This induces a map $q \mapsto p=P(q)$ which is easily seen to be a diffeomorphism using Proposition 2.2.

Conversely if there is a map $P(\cdot)$ with the properties of the proposition and $p \in N$, let $q=\exp (\ell(\eta(p)) \eta(x))$ be the point where the geodesic $\gamma$ normal to $N$ at $p$ meets $N_{+}$. As $P(\cdot)$ is bijective there is a point $q_{1} \in N_{+}$so that $p=P\left(q_{1}\right)$. Since $M$ is globally hyperbolic there is a maximizing geodesic $\gamma_{1}$ from $p$ to $q_{1}$ and by the properties of $P(\cdot)$ this geodesic must also maximize the distance of its point from $N$. Thus $\gamma_{1}$ will be normal to $N$ at $p$ so that $\gamma_{1}=\gamma$, and $q_{1}=q$. Thus $\gamma(t)=\exp (t \eta(p))$ maximizes distances from $N$ for $t \in[0, \ell(\eta(p))]$ which implies 
that none of the points $\gamma(t)$ is a cut point of $N$ for $0 \leq t<\ell(\eta(p))$. To see that the point $q=\exp (\ell(\eta(p)) \eta(x))$ cannot be a cut point note that because $P$ is bijective for any $q \in N_{+}$there is at most one maximizing geodesic from $q$ to $N$ and therefore the only way that $q$ can be a cut point is if $q$ is a focal point of $N$, that is a point where the derivative of the exponential on the normal bundle to $N$ is not injective. But the restriction of the exponential to the hypersurface $\mathcal{N}_{+}:=\{(\ell(\eta(p)), p): p \in N\}$ is essentially just the map $P^{-1}$ and thus has an injective differential. For a fixed $p \in N$ the curve $t \mapsto(t, p)$ in the normal bundle maps to $t \mapsto \exp (t \eta(p))$ which has a nonzero tangent vector for all $t$. As $t \mapsto(t, p)$ is transverse to the hypersurface $\mathcal{N}_{+}$this implies that exp has injective differential at all points of $\mathcal{N}_{+}$which shows that no point of $N_{+}$is a focal point.

Proof of 2.4. Let $\mathcal{H}_{p}=\left\{(v, t): v \in H_{p}, t \in[0, \ell(v)]\right\}$. Then if $(M, g)$ is SGM, the map $(v, t) \mapsto \exp (t v)$ is a diffeomorphism of $\mathcal{M}$ with $I^{+}(p)$. If $V \in T(N)_{p}$, there is a unique $\theta \geq 0$ so that $v:=\cosh (\theta) \eta(p)+V$ is a future pointing time-like unit vector. Let $q$ be the point where the geodesic $\gamma(t):=\exp (t v)$ meets $N_{+}$. Then as $\gamma(t)$ is free of cut points $\nabla_{p} d^{\partial}(\cdot, q)$ is the orthogonal projection of $\gamma^{\prime}(0)$ onto $T(N)_{p}$. That is $\nabla_{p} d^{\partial}(\cdot, q)=V$. As the point $q$ is the point where the geodesic determined by $\cosh (\theta) \eta(p)+V$ meets $N_{+}$, the point $q$ is the unique point with $\nabla_{p} d^{\partial}(\cdot, q)=V$. Now using the diffeomorphism of Proposition 2.1 it follows that the map $q \rightarrow \nabla_{p} d^{\partial}(\cdot, q)$ is a diffeomorphism.

Conversely if there is a time-like segment $\gamma:[0, L] \rightarrow M$ which is not strongly maximizing, then by extending it to a maximal geodesic segment it can be assumed that for some $p \in N$ and $v \in H_{p}$ that $\gamma(t)=\exp (t v)$ is defined on $[0, \ell(v)]$. Let $\gamma\left(t_{0}\right)$ be the first cut point of $p=\gamma(0)$ along $\gamma$ and let $q=\gamma(\ell(v))$ be the point where $\gamma$ meets $N_{+}$. If $t_{0}<\ell(v)$ then $\gamma$ does not maximize the distance from $p$ to $q$. Write $v=\cosh (\theta) \eta+V$ where $\theta \geq 0$ and $V \in T(N)_{p}$. As $v$ is a unit vector $\|V\|=\sinh (\theta)$ so that $V$ determines $\theta$. Then there cannot be any $q_{1} \in I^{+}(p) \cap N_{+}$ so that $\nabla_{p} d^{\partial}\left(\cdot, q_{1}\right)=V$, for then the maximizing geodesic from $p$ to $q_{1}$ would have initial vector $v=\cosh (\theta) \eta+V$ which in turn implies $q_{1}=\exp (\ell(v))=q$. This contradicts $\gamma$ not being maximizing. This leaves the case $t_{0}=\ell(v)$ which splits into two sub-cases. The first being that $q=\exp (\ell(v) v)$ is a conjugate point of $p$, and the second being that $q$ is not a conjugate point, but there are two or more maximizing geodesics from $p$ to $q$. To rule out the first sub-case note that as the mapping $q \mapsto \nabla_{p} d^{\partial}(\cdot, q)$ is a diffeomorphism of $I^{+}(p) \cap N_{+}$with $H_{p}$, the derivative of the exponential map is non-singular at any of the points $\ell(u) u$ with $u \in H_{p}$. Thus $q=\exp (\ell(v) v)$ is not a conjugate point of $p$. This leaves the case where there are two maximizing geodesics $\gamma(t)=\exp (t v)$ and $\gamma_{1}(t)=\exp \left(t v_{1}\right)$ from $p$ to $q$. Write $\gamma^{\prime}(0)=\cosh (\theta) \eta+V$ and $\gamma_{1}^{\prime}(0)=\cosh \left(\theta_{1}\right)+V_{1}$ where $V, V_{1} \in T(N)_{p}$. But as there are no conjugate points along the geodesics $\gamma$ and $\gamma_{1}$ we have $V_{1}=\nabla_{p} d^{\partial}(\cdot, q)=V$. As $V$ and $V_{1}$ determine $\theta$ and $\theta_{1}$ this implies $\gamma^{\prime}(0)=\gamma_{1}^{\prime}(0)$ which implies $\gamma=\gamma_{1}$, contradicting $\gamma \neq \gamma_{1}$. This completes the proof of the characterization of SGM in terms of boundary distances.

If $(M, g)$ is SGM, then for each $p \in N, v \in T(N)_{p}$. Write $v=\cosh (\theta) \eta+V$ where $\theta \geq 0$ and $V \in T(N)_{p}$. Let $q \in N_{+}$be the unique point so that $\nabla_{p} d^{\partial}(\cdot, q)=V$. Then $\ell(v)=d^{\partial}(p, q)$ and $q=\exp (\ell(v) v)$. The velocity vector of $\gamma(t)=\exp (t v)$ at $t=\ell(v)$ is $\nabla_{q} d^{\partial}(p, \cdot)+\cosh (\theta) \xi$ where $\xi$ is the future pointing unit vector to $N_{+}$at $q$. This shows that when $(M, g)$ is SGM, the length of the geodesic $\gamma(t u)=\exp (t u)$, the point where this geodesic meets $N_{+}$and its velocity vector at $N_{+}$can all be 


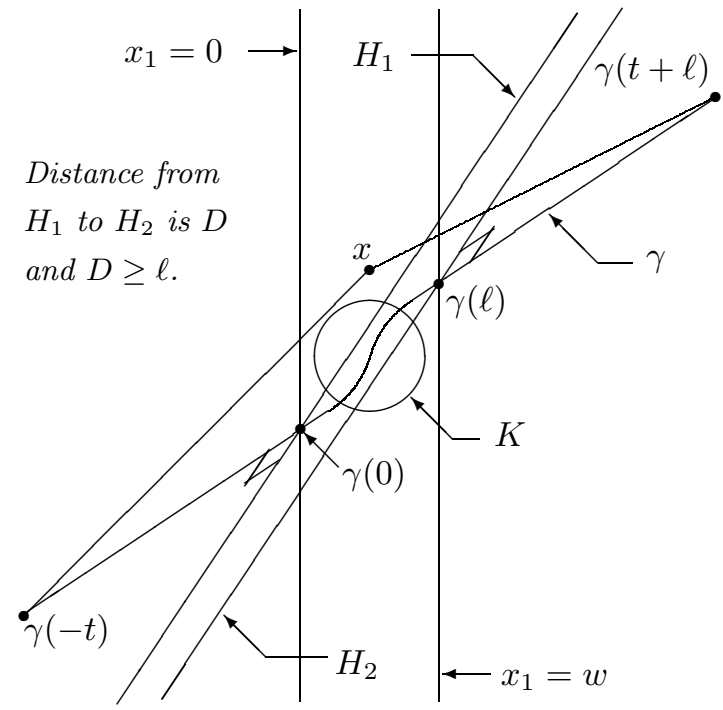

Figure 1

determined by $d^{\partial}$. Thus any $\left(M^{*}, g^{*}\right)$ boundary isometric to $(M, g)$ will also be lens equivalent to $(M, g)$.

Proof of Proposition 2.5. We adapt Croke's argument [5, §4] to the Lorentzian setting. Assume $g$ is a Lorentzian metric that agrees with the usual Lorentzian metric $g_{0}=-d x_{1}^{2}+d x_{2}^{2}+\cdots+d x_{n}^{2}$ outside of the compact set $K \subset \mathbb{R}^{n}[0, w]:=\{0 \leq$ $\left.x_{1} \leq w\right\}$ and that $\left(\mathbb{R}^{n}, g\right)$ is globally hyperbolic and future one connected. Then let $p, q \in \mathbb{R}^{n}$ with $p$ in the future of $q$. Then $[1, \S 9.1]$ there is exactly one time-like geodesic of $\left(\mathbb{R}^{n}, g\right)$ through $p$ and $q$ and the unique geodesic segment between $p$ and $q$ maximizes length. This implies that if $\gamma$ is a future directed time-like geodesic of $\left(\mathbb{R}^{n}, g\right)$ then the direction it enters $\mathbb{R}^{n}[0, w]$ and the direction it leaves $\mathbb{R}^{n}[0, w]$ are the same. For if not there would be a time-like geodesic $\gamma_{1}$ that misses $K$ and meets $\gamma$ in two points.

Let $N:=\left\{x_{1}=0\right\}$ and $N_{+}:=\left\{x_{1}=w\right\}$ be the past and future boundaries of $\mathbb{R}^{n}[0, w], d^{\partial}: N \times N_{+} \rightarrow[0, \infty)$ the boundary distance function of $g$ and $d_{0}^{\partial}$, also defined on $N \times N_{+}$, the boundary distance function of $g_{0}$. We now show $d^{\partial}=d_{0}^{\partial}$. Let $p \in N$ and $q \in N_{+}$and let $\gamma(t)$ be a time-like future pointing unit speed geodesic that misses the compact set $K$ but is parallel to the segment from $p$ to $q$. Then $\gamma$ is a geodesic for both the metrics $g$ and $g_{0}$. Letting $d$ and $d_{0}$ be the distance functions of $g$ and $g_{0}$ we have from the reverse triangle inequality for large $t$

$$
\begin{aligned}
2 t & =d_{0}(\gamma(-t), \gamma(t))=d(\gamma(-t), \gamma(t)) \\
& \geq d(\gamma(-t), p)+d(p, q)+d(q, \gamma(t))=d_{0}(\gamma(-t), p)+d^{\partial}(p, q)+d_{0}(q, \gamma(t)) .
\end{aligned}
$$

Thus $d_{0}^{\partial}(p, q)=\lim _{t \rightarrow \infty}\left[2 t-\left(d_{0}(\gamma(-t), p)+d(q, \gamma(t))\right)\right] \geq d^{\partial}(p, q)$.

To get the inequality $d_{0}^{\partial}(p, q) \leq d^{\partial}(p, q)$ in the case $p \in\left\{x_{1}=0\right\}, q \in\left\{x_{1}=w\right\}$ and $q \in I^{+}(p)$ let $\gamma$ be the time-like geodesic of the metric $g$ that passes through $p$ and $q$.

Parameterize $\gamma$ so that $\gamma(0)=p$ and let $\ell=d^{\partial}(p, q)$ be the number so that 
$\gamma(\ell)=q$. The restriction of $\gamma$ to each of the intervals $(-\infty, 0)$ and $(\ell, \infty)$ will be straight lines as the metrics agree outside of $K$. Let $H_{1}$ be the space-like hyperplane through $p=\gamma(0)$ and orthogonal (with respect to $g_{0}$ ) to $\gamma, H_{2}$ be the hyperplane through $q=\gamma(\ell)$ orthogonal to $\gamma$ and let $D$ be the distance between $H_{1}$ and $H_{2}$ (see Figure 1). By the reverse triangle inequality $D \geq d(p, q)=\ell$. Choose a point $x$ that is not causally related to any point in $K$ and connect it to the points $\gamma(-t)$ and $\gamma(t+\ell)$ by line segments. By the reverse triangle inequality

$$
\begin{aligned}
2 t+\ell & =d(\gamma(-t), \gamma(t+\ell)) \\
& \geq d(\gamma(-t), x)+d(x, \gamma(t+\ell)) .
\end{aligned}
$$

Therefore $d^{\partial}(p, q)=\ell \geq d_{0}(\gamma(-t), x)+d_{0}(x, \gamma(t+\ell))-2 t$. But it is easy to check that $\lim _{t \rightarrow \infty} d_{0}(\gamma(-t), x)+d_{0}(x, \gamma(t+\ell))-2 t=D \geq d_{0}(p, q)$. This yields $d^{\partial}(p, q) \geq d_{0}(p, q)$ and completes the proof $\left(\mathbb{R}^{n}[0, w], g\right)$ is boundary isometric to $\left(\mathbb{R}^{n}[0, w], g_{0}\right)$. As $\left(\mathbb{R}^{n}[0, w], g_{0}\right)$ is clearly SGM, Proposition 2.4 implies $\left(\mathbb{R}^{n}[0, w], g\right)$ and $\left(\mathbb{R}^{n}[0, w], g_{0}\right)$ are lens equivalent.

\section{Boundary Distances and Conformal Type}

Let $(M, g)$ be a two dimensional space-like slab. Part of the definition of spacelike slab is that every inextendible causal curve meets both the past boundary $N$ and the future boundary $N_{+}$. It follows that each of the two null geodesics through a point will meet both $N$ and $N_{+}$. Since $(M, g)$ is time oriented it is possible to choose two foliations of $M$ by the null directions. Let $\mathcal{L}_{-}$be the foliation by null geodesics so that if a person stands on $N$ and looks into the future, then the curves of $\mathcal{L}_{-}$move to the right. Likewise let $\mathcal{L}_{+}$be the foliation by null geodesics so that when standing on $N$ and looking to the future the elements of $\mathcal{L}_{+}$move to the left. As $(M, g)$ is globally hyperbolic and $N$ is a Cauchy hypersurface, $M$ is diffeomorphic to $N \times[0,1]$ (cf. [1, §2.2]) and as $N$ is one dimensional it is isometric to either $\mathbb{R}$ or the circle $S^{1}$. Thus if $N$ is simply connected, then $N$ is diffeomorphic to $\mathbb{R}$ and as the past boundary of a space-like slab is complete, the past boundary of a simply connected two dimensional surface is isometric to $\mathbb{R}$.

Now assume that $(M, g)$ is a simply connected two dimensional space-like slab. Fix a point $p_{0}$ of the past boundary $N$ and let $c: \mathbb{R} \rightarrow N$ be the unit speed parameterization of $N$ so that for increasing $s$, the point $c(s)$ moves to the left compared to someone standing on $N$ and looking to the future. If $M$ is simply connected then there are unique functions $u$ and $v$ defined on $M$ by the differential equations

$$
\left\{\begin{array}{l}
u(c(s))=s, \quad \text { and } d u(X)=0 \text { if } X \text { is tangent to } \mathcal{L}_{-}, \\
v(c(s))=s, \quad \text { and } d v(X)=0 \text { if } X \text { is tangent to } \mathcal{L}_{+} .
\end{array}\right.
$$

From this it follows for all $q \in M$ that

$$
u(q)-v(q) \geq 0 \quad \text { with equality iff } q \in N .
$$

The function $u$ is constant on the curves of the foliations $\mathcal{L}_{-}$and $v$ is constant on the curves of the foliation $\mathcal{L}_{+}$. For a point $q$ the boundary of the chronological past $I^{-}(q)$ of $q$ is the union of the two null curves through $q$. As the chronological past is also given by $I^{-}(q)=\{p: d(p, q)>0\}$ it follows that there are the formulas

$$
\left\{\begin{array}{l}
u(q)=\sup \{s: d(c(s), q)>0\} \\
v(q)=\inf \{s: d(c(s), q)>0\}
\end{array}\right.
$$




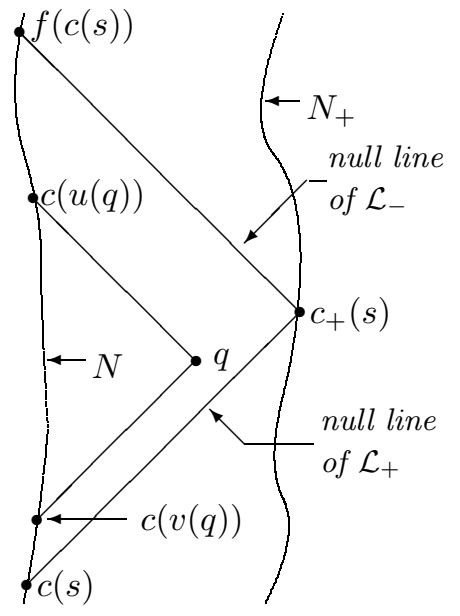

Figure 2

for $u$ and $v$ in terms of distance function. Define a map $\varphi: M \rightarrow \mathbb{R}^{2}$ by

$$
\varphi(p):=(u(p)-v(p), u(p)+v(p)) .
$$

If $\mathbb{R}^{2}$ has the Lorentzian metric $g_{0}=-d x^{2}+d y^{2}$, then $\varphi$ sends null curves of $(M, g)$ to null curves of $\left(\mathbb{R}^{2}, g_{0}\right)$ and therefore $\varphi$ is conformal. Points on $N$ are of the form $c(s)$ and thus $\varphi(c(s))=(0,2 s)$ and it follows that $\varphi$ maps $N$ bijectively onto the $y$-axis. We wish to show that the image of $N_{+}$can be defined in terms of boundary distances. In what follows we will take the terms "left" and "right" to mean the left or right for someone on $M$ facing to the future. For the point $c(s)$ on $N$ let $c_{+}(s)$ be the point on $N_{+}$where the null curve from $\mathcal{L}_{+}$and passing through $c(s)$ meets $N_{+}$(see Figure 2). This can be defined in terms of boundary distances as

$$
c_{+}(s)=\text { left endpoint of the interval }\left\{q \in N_{+}: d^{\partial}(c(s), q)>0\right\}
$$

and then define $f(c(s))$ to be the point where the null line from $\mathcal{L}_{-}$and through $c_{+}(s)$ intersects $N$. That is

$$
f(c(s))=\text { left endpoint of the interval }\left\{p \in N: d^{\partial}\left(p, c_{+}(s)\right)>0\right\}
$$

The map $s \mapsto c_{+}(s)$ is a diffeomorphism of $\mathbb{R}$ with $N_{+}$. Note that from the definition of $v, c_{+}(s)$ and $f\left(c_{+}(s)\right)$ we have $v\left(c_{+}(s)\right)=s, u\left(c_{+}(s)\right)=u(f(c(s)))$ and thus

$$
\varphi\left(c_{+}(s)\right)=(u(f(c(s)))-s, u(f(c(s)))+s) .
$$

The formulas (1), (2) and (3) show that $\varphi\left(c_{+}(s)\right)$ is determined by the function $d^{\partial}: N \times N_{+} \rightarrow(0, \infty)$. Thus $\varphi[M]$ is the region of the plane between the $y$-axis and the curve $s \mapsto \varphi\left(c_{+}(s)\right)$ and so the above discussion along with Proposition 2.4 yields

Proposition 3.1. Let $(M, g)$ be a simply connected two dimensional space-like slab. If $\left(M^{*}, g^{*}\right)$ is boundary isometric to $(M, g)$ then there is a unique conformal diffeomorphism $\Phi:(M, g) \rightarrow\left(M^{*}, g^{*}\right)$ that extends the isometry between the boundaries. 


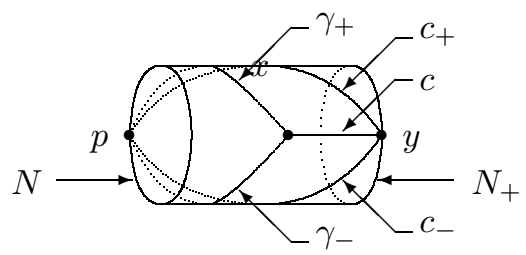

Figure 3

It is natural to try to reduce the general case (i.e. where the surface $(M, g)$ is not simply connected) to the last proposition by passing to the universal cover. However it is not clear in general that if $(M, g)$ and $\left(M^{*}, g^{*}\right)$ are boundary isometric that their universal covers are boundary isometric. This motivates

Definition 3.2. A space-like slab $(M, g)$ has simply connected boundary cones iff for all $p \in N$ (the past boundary) and $q \in N_{+}$(the future boundary) the chronological future $I^{+}(p)$ of $p$ and the chronological past $I^{-}(q)$ are simply connected.

As an example let $S^{1}$ be the unit circle and let $(M, g)=\left([0, w] \times S^{1},-d t^{2}+d \theta^{2}\right)$. Then $(M, g)$ has simply connected boundary cones if and only if $w \leq \pi$. In two dimensions a space-like slab with past boundary $N$ is diffeomorphic to $[0,1] \times N$. Thus in two dimensions when $M$ is not simply connected, $N$ is a circle and $M$ is diffeomorphic to a cylinder.

Assume that $M$ is two dimensional and not simply connected. Let $p \in N$ and let $M^{\circ}:=M \backslash \partial M$ be the interior of $M$. Let $\gamma_{+}$and $\gamma_{-}$be the two null curves through $p$. Then as $p$ is on the past boundary we see that near $p$ the boundary of $I^{+}(p)$ is the union $\gamma_{+}$and $\gamma_{-}$. If $\gamma_{+}$and $\gamma_{-}$do not intersect at a point in $M^{\circ}$, then $I^{+}(p)$ will be simply connected.

If $\gamma_{+}$do intersect in $M^{\circ}$ at some point $x$ then there is a time-like curve $c$ connecting $x$ to some point $y$ in the future boundary $N_{+}$(see Figure 3). By smoothing the curve that consists of following $\gamma_{+}$from $p$ to $x$ and then following $c$ from $x$ to $y$ to get a time-like curve $c_{+}$from $p$ to $y$. Likewise there is another timelike curve $c_{-}$ from $p$ to $y$ that approximates following $\gamma_{-}$from $p$ to $x$ and then following $c$ from $x$ to $y$. Then the union of the curves $c_{+}$and $c_{-}$is a loop in $I^{+}(p)$ that cannot be contracted and therefore $I^{+}(p)$ is not simply connected.

Proposition 3.3. 1. Let $(M, g)$ be a two dimensional space-like slab and $p_{0} \in N$ and $q_{0} \in N_{+}$. Then $I^{+}\left(p_{0}\right)$ is simply connected if and only if $\left\{q \in N_{+}: d^{\partial}\left(p_{0}, q\right)=\right.$ $0\} \neq \emptyset$ and $I^{-}\left(q_{0}\right)$ is simply connected if and only if $\left\{p \in N: d^{\partial}\left(p, q_{0}\right)=0\right\} \neq \emptyset$. Thus if $(M, g)$ is a two dimensional space-like slab with simply connected boundary cones, and $\left(M^{*}, g^{*}\right)$ is boundary isometric to $(M, g)$, then $\left(M^{*}, g^{*}\right)$ also has simply connected boundary cones.

2. If $(M, g)$ is a two dimensional SGM space-like slab, then $(M, g)$ has simply connected boundary cones.

Proof. To prove the first part note that in the notation above, $\left\{q \in N_{+}: d^{\partial}\left(p_{0}, q\right)=\right.$ $0\} \neq \emptyset$ if and only if the two curves $\gamma_{+}$and $\gamma_{-}$intersect in $M^{\circ}$ and the result follows from the preceding discussion. To prove the second part we show that if one of the sets $I^{+}(p)$ for $p \in N$ is not simply connected, then $(M, g)$ is not SGM. As above if $I^{+}(p)$ is not simply connected there is a point $y \in N_{+}$that can be connected to $x$ by two time-like curves $c_{-}$and $c_{+}$that are not homotopic. Because $(M, g)$ 
is globally hyperbolic there will be a maximizing geodesic in the homotopy class (with endpoints fixed) of each of the curves $c_{-}$and $c_{+}$so we can assume that each of $c_{-}$and $c_{+}$is a geodesic segment from $p$ to $y$. But then either $y$ is a cut point of $p$ along both $c_{-}$and $c_{+}$or at least one of $c_{-}$or $c_{+}$contains an interior cut point of $p$. In either case the definition of SGM is violated.

Proposition 3.4. Let $(M, g)$ and $\left(M^{*}, g^{*}\right)$ be space-like slabs which are boundary isometric and which have simply connected boundary cones. Then the universal covers of $(M, g)$ and $\left(M^{*}, g^{*}\right)$ are boundary isometric.

Proof. A tedious, but more or less straightforward exercise.

Theorem 3.5. Let $(M, g)$ be a two dimensional space-like slab with simply connected boundary cones (for example if $(M, g)$ is simply connected or SGM). Let $\left(M^{*}, g^{*}\right)$ be boundary isometric to $(M, g)$. Then $\left(M^{*}, g^{*}\right)$ also has simply connected boundary cones and the boundary isometry between $\partial M$ and $\partial M^{*}$ extends to a unique conformal diffeomorphism between $(M, g)$ and $\left(M^{*}, g^{*}\right)$.

Proof. The case of $(M, g)$ simply connected was covered by Proposition 3.1. If $(M, g)$ is not simply connected then let $(\widehat{M}, \widehat{g})$ and $\left(\widehat{M}^{*}, \widehat{g}^{*}\right)$ be the universal covering spaces of $(M, g)$ and $\left(M^{*}, g^{*}\right)$. Let $\widehat{\varphi}: \partial \widehat{M} \rightarrow \partial \widehat{M}^{*}$ be the lift of the boundary isometry given by Proposition 3.4 and let $\widehat{\Phi}: \widehat{M} \rightarrow \widehat{M}^{*}$ be the unique conformal extension of $\widehat{\varphi}$. Let $G$ be the group of deck transformations of $\pi: \widehat{M} \rightarrow M$ and let $G^{*}$ be the group of deck transformations of $\pi^{*}: \widehat{M}^{*} \rightarrow M^{*}$. Because $\widehat{\Phi}$ is determined by its boundary values there is an isomorphism $\rho: G \rightarrow G^{*}$ so that $\rho(a) \circ \widehat{\Phi}=\widehat{\Phi} \circ a$ for all $a \in G$. This implies the map $\Phi: M \rightarrow M^{*}$ given by $\Phi(\pi(x))=\pi^{*}(\widehat{\Phi}(x))$ is well defined and is a conformal diffeomorphism from $(M, g)$ to $\left(M^{*}, g^{*}\right)$ extending the boundary isometry. If $\Phi, \Psi: M \rightarrow M^{*}$ are both conformal diffeomorphisms that extend the boundary isometry, the map $\Phi^{-1} \circ \Psi: M \rightarrow M$ is a conformal automorphism which is the identity on $\partial M$. This implies $\Phi^{-1} \circ \Psi=\operatorname{Id}_{M}$ which proves uniqueness.

3.1. Examples. We now give examples of Lorentzian surfaces that are boundary isometric but not isometric. These examples are the Lorentzian versions of the examples in $[5, \S 2]$; however we work with locally conformally flat metrics instead of surfaces of revolution. Let $w>1$ and let $M$ be the strip $\{(x, y): 0 \leq x \leq w\}$ in the plane $\mathbb{R}^{2}$. Let $\psi(x)$ be a function defined on the line $-\infty<x<\infty$ so that the support of $\psi$ is in the interval $[0,1]$ and so that $0 \leq \psi<1$. For each $\delta$ with $0<\delta<w-1$, set $E_{\delta}(x)=1-\psi(x-\delta)$ and let $g_{\delta}=E_{\delta}(x)^{2}\left(-d x^{2}+d y^{2}\right)$. Every time-like curve is of the form $y=u(x)$ where $\left|u^{\prime}(x)\right|<1$. The length of such a curve is $\int_{0}^{L} E_{\delta}(x) \sqrt{1-u^{\prime}(x)^{2}} d x$ and therefore the Euler-Lagrange equation for geodesics is $\frac{d}{d x}\left(E_{\delta}(x) u^{\prime}(x) / \sqrt{1-u^{\prime}(x)^{2}}\right)=0$. Integrating with respect to $x$ and solving for $u^{\prime}(x)$ shows that for the constant $a=u^{\prime}(0) / \sqrt{1-u^{\prime}(0)^{2}}$

$$
u^{\prime}(x)=\frac{a}{\sqrt{E_{\delta}(x)^{2}+a^{2}}}, \quad u(x)=u(0)+\int_{0}^{x} \frac{a d t}{\sqrt{E_{\delta}(t)^{2}+a^{2}}} .
$$

This implies that the values of $u(w)$ and $u^{\prime}(w)$ only depend on $u(0)$ and $u^{\prime}(0)$, but are independent of $\delta \in(0, w-1)$. Thus all the manifolds $\left(M, g_{\delta}\right)$ are lens equivalent, but if $\psi \neq \equiv 0$ no two of them are isometric. To get compact examples identify the edge line $y=0$ with the line $y=b$ to get lens equivalent metrics on a cylinder. 
As $E_{\delta}(x) \leq 1$ it follows that any time-like geodesic $y=u(x)$ will have length $\leq w \sqrt{1-u^{\prime}(0)^{2}} \leq w$. Let $K(x)=E_{\delta}^{-2}(x)\left(\log \left(E_{\delta}(x)\right)\right)^{\prime \prime}$ be the Gaussian curvature of $g_{\delta}$ and let $\gamma(t)$ be a unit speed time-like geodesic of $\left(M, g_{\delta}\right)$. If $e(t)$ is a parallel normal field along $\gamma$, then the Jacobi equation for the field $X(t):=y(t) e(t)$ is $y^{\prime \prime}+K(\gamma(t)) y(t)=0$. Thus by basic comparison theory for ordinary differential equations we see that if $|K(x)| \leq \pi^{2} / w^{2}$, then no time-like geodesic will have any conjugate points and as $\left(M, g_{\delta}\right)$ is globally hyperbolic this implies that all geodesics of $\left(M, g_{\delta}\right)$ maximize between all of their points (cf. $\left.[1, \S 9.1]\right)$ and so $|K(x)| \leq \pi^{2} / w^{2}$ implies that $\left(M, g_{\delta}\right)$ is SGM. If $|K(x)|<\left(4 \pi^{2}\right)$, then the past boundary $N$ will have no focal points, which implies that $\left(M, g_{\delta}\right)$ is free of cut points. Finally to get compact examples identify the line $y=0$ with the line $y=b$ to get a cylinder which we denote by $M_{b}$. Then the conditions

$$
2 w<b, \quad\left\|E_{\delta}^{-2}\left(\log E_{\delta}\right)^{\prime \prime}\right\|_{L^{\infty}}<\frac{\pi^{2}}{4 w^{2}}
$$

imply that the space-like slabs $\left(M_{b}, g_{\delta}\right)$ are all SGM, all free of cut points and all are boundary isometric. They also all have the same conformal type and the same area. But if $\psi \not \equiv 0$ then no two of them are isometric.

\section{Volume Estimates and Boundary Distances}

Let $(M, g)$ be a space-like slab with past boundary $N$ and let $H(M), H(N), \zeta^{t}$, and $\ell(v)$ be as in Section 2 .

Definition 4.1. The two space-like slabs $(M, g)$ and $\left(M^{*}, g^{*}\right)$ are length equivalent if and only if there is an isometry $\varphi: N \rightarrow N^{*}$ so that for all $v \in H(N)$ there holds $\ell\left(\varphi_{\#} v\right)=\ell(v)$, where $\varphi_{\#}$ is as in Section 1 .

If $(M, g)$ and $\left(M^{*}, g^{*}\right)$ are lens equivalent then they are length equivalent.

Theorem 4.2. Let $(M, g)$ and $\left(M^{*}, g^{*}\right)$ be compact two dimensional space-like slabs which are free of cut points. Assume that for some $w>0$ there is a bound

$$
\ell(v) \leq \frac{w}{|\langle v, \eta\rangle|}
$$

for all $v \in H(N)$. Then if $(M, g)$ and $\left(M^{*}, g^{*}\right)$ are length equivalent, they have the same area. In particular if $\ell(v)=w /|\langle v, \eta\rangle|$ for all $v$ then $\operatorname{Area}(M)=w \operatorname{Length}(N)$.

We will set up the analysis for this result in general dimensions as this does lead to a bound on the volume of a Lorentzian manifold with boundary.

Theorem 4.3. Let $(M, g)$ be a compact space-like slab which is free of cut points and so that the bound (4) holds. For each $r \in[0, w]$ let $N_{r}:=\left\{\exp _{x}(r \eta(x))\right.$ : $x \in N\}$ ( $\eta$ is the inward unit normal field along $N$ ) be the hypersurface parallel to $N=N_{0}$ at a distance $r$. Let $\lambda_{1}, \ldots, \lambda_{n-1}$ be the principal curvatures of $N_{r}$ and let $B:=2 \max \left\{\left|\lambda_{i}(x)\right|: 1 \leq i \leq n-1, r \in[0, w], x \in N_{r}\right\}$. Then there is a constant $C(n)$ depending only on the dimension so that

$$
\operatorname{Vol}(M) \leq w \operatorname{Area}(N)\left(1+C(n)\left(\frac{2+B w}{2}\right)^{n+1} B w\right) .
$$

When $n=2$ the constant $C(2)=0$ so this reduces to $\operatorname{Area}(M) \leq w \operatorname{Length}(N)$. 
Remark 4.4. Let $(M, g)$ be a general $n$-dimensional compact space-like slab which is free of cut points and let $(n-1) H=\lambda_{1}+\cdots+\lambda_{n-1}$ be the mean curvature of the parallel hypersurfaces $N_{r}$. If for all $p \in N$ the inequality $\ell(\eta(p)) \leq w$ holds and the mean curvature is bounded, say $|H| \leq B$, then the first variation formula implies the bound

$\operatorname{Vol}(M) \leq \operatorname{Area}(N) \int_{0}^{w} e^{(n-1) B t} d t=\operatorname{Area}(N) \frac{e^{(n-1) B w}-1}{(n-1) B w}$.

Our basic tool is the Lorentzian version of Santaló's formula which we now state. Each fiber $H_{x}$ of the bundle $H(M)$ has a natural measure, the area measure $d u$ as the hyperbolic space $H_{y}=\mathbf{H}^{n-1}$. Thus the total space of the bundle $H(M)$ has the local product measure $d u d y$ where $d y$ is the volume measure on $M$. Likewise $H(N)$ has the local product measure $d v d x$ where $d v$ is the area measure on the fibers $H_{x}=\mathbf{H}^{n-1}$ and $d x$ is the area measure on $N$.

Theorem 4.5 (Santaló's Formula). If $(M, g)$ is a space-like slab, then for any function $f$ defined on $H(M)$

$$
\int_{M} \int_{H_{y}} f(u) d u d y=\int_{N} \int_{H_{x}} \int_{0}^{\ell(v)} f\left(\zeta^{t} v\right) d t|\langle v, \eta(x)\rangle| d v d x .
$$

(Here $\langle\rangle=,g($,$) and \zeta^{t}$ is the geodesic flow.)

For a brief discussion of the proof see the appendix.

Call a function $f$ defined on $H(M)$ adapted iff $f \geq 0$ and for all $y \in M$

$$
\int_{H_{y}} f(u) d u=1 .
$$

Lemma 4.6. If $(M, g)$ is a compact space-like slab, then for any adapted function $f$ on $H(M)$

$$
\begin{aligned}
\operatorname{Vol}(M)-\int_{N} \int_{H_{x}} f(v) \mid\langle v & , \eta(x)\rangle \mid \ell(v) d v d x \\
& =\int_{N} \int_{H_{x}} \int_{0}^{\ell(v)}\left(f\left(\zeta^{t} v\right)-f(v)\right) d t|\langle v, \eta(x)\rangle| d v d x .
\end{aligned}
$$

In the special case that $\ell(v) \equiv w /|\langle v, \eta\rangle|$ this reduces to

$$
\operatorname{Vol}(M)-w \operatorname{Area}(N)=\int_{N} \int_{H_{x}} \int_{0}^{\ell(v)}\left(f\left(\zeta^{t} v\right)-f(v)\right) d t|\langle v, \eta(x)\rangle| d v d x,
$$

so in this case the integral on the right is independent of which adapted function is used.

Proof. We use Santaló's formula and the relation (7).

$$
\begin{aligned}
\operatorname{Vol}(M)= & \int_{M} \int_{H_{y}} f(u) d u d y=\int_{N} \int_{H_{x}} \int_{0}^{\ell(v)} f\left(\zeta^{t} v\right) d t|\langle v, \eta(x)\rangle| d v d x \\
= & \int_{N} \int_{H_{x}} f(v) \ell(v)|\langle v, \eta(x)\rangle| d v d x \\
& +\int_{N} \int_{H_{x}} \int_{0}^{\ell(v)}\left(f\left(\zeta^{t} v\right)-f(v)\right) d t|\langle v, \eta(x)\rangle| d v d x .
\end{aligned}
$$


If $\ell(v)=w /|\langle v, \eta\rangle|$ then, using $\int_{H_{x}} f(v) d v=1$,

$$
\int_{N} \int_{H_{x}} f(v) \ell(v)|\langle v, \eta(x)\rangle| d v d x=w \int_{N} \int_{H_{x}} f(v) d v d x=w \operatorname{Area}(N)
$$

Now assume that $(M, g)$ is free of cut points and compact. By Proposition 2.2 the map $(x, t) \mapsto \exp (t \eta(x))$ is a diffeomorphism. Thus in this case we can define a forward pointing unit vector field $\mathbf{n}$ on $M$ by

$$
\mathbf{n}(\exp (t \eta(x))):=\left.\frac{d}{d s} \exp (s \eta(x))\right|_{s=t} .
$$

Note that the flow lines of the vector field $\mathbf{n}$ are the curves $\exp (t \eta(x))$ which are geodesics. Thus $\nabla_{\mathbf{n}} \mathbf{n} \equiv 0$. Let $v \in H(N)$. We now estimate the angle that $\zeta^{t} v$ makes with $\mathbf{n}$. For $u \in H_{y}$ let $\rho(u)$ be the distance in $H_{y}$ from $\mathbf{n}(y)$. That is $\rho(u)$ is the hyperbolic angle that $u$ makes with the direction $\mathbf{n}(y)$. Let $\gamma(t):=\exp (t v)$ and let and $X(t)$ be the unit vector field along $\gamma(t)$ that is normal to $\mathbf{n}$ and so that if $\rho_{t}:=\rho\left(\gamma^{\prime}(t)\right)$ then

$$
\gamma^{\prime}(t)=\cosh \left(\rho_{t}\right) \mathbf{n}+\sinh \left(\rho_{t}\right) X(t) .
$$

Define $y(t):=\cosh \left(\rho_{t}\right)$. Then the last equation is

$$
\gamma^{\prime}(t)=y(t) \mathbf{n}+\sqrt{y(t)^{2}-1} X(t) .
$$

As $\gamma$ is a geodesic

$$
0=\nabla_{\gamma^{\prime}(t)} \gamma^{\prime}(t)=y^{\prime}(t) \mathbf{n}+y(t) \nabla_{\gamma^{\prime}(t)} \mathbf{n}+\frac{y(t) y^{\prime}(t)}{\sqrt{y(t)^{2}-1}} X+\sqrt{y(t)^{2}-1} \nabla_{\gamma^{\prime}(t)} X .
$$

Using that $\mathbf{n} \perp X, \mathbf{n} \perp \nabla_{\gamma^{\prime}} \mathbf{n}$, take the inner product of the last equation with $\mathbf{n}$

$$
\begin{aligned}
y^{\prime}(t) & =\sqrt{y(t)^{2}-1}\left\langle\nabla_{\gamma^{\prime}(t)} X, \mathbf{n}\right\rangle=-\sqrt{y(t)^{2}-1}\left\langle X, \nabla_{\gamma^{\prime}(t)} \mathbf{n}\right\rangle \\
& =-\sqrt{y(t)^{2}-1}\left\langle X, \nabla_{\left(y(t) \mathbf{n}+\sqrt{y(t)^{2}-1} X\right)} \mathbf{n}\right\rangle \\
& =-\sqrt{y(t)^{2}-1}\left\langle X, \nabla_{\sqrt{y(t)^{2}-1} X} \mathbf{n}\right\rangle \quad\left(\text { as } \nabla_{\mathbf{n}} \mathbf{n}=0\right) \\
& =-\left(y(t)^{2}-1\right)\left\langle X, \nabla_{X} \mathbf{n}\right\rangle .
\end{aligned}
$$

By compactness and continuity there is a constant $B>0$ so that for all unit vectors $X \perp \mathbf{n}$ the inequality $\left|\left\langle X, \nabla_{X} \mathbf{n}\right\rangle\right| \leq B / 2$ holds. To be more precise let $\lambda_{1}, \ldots, \lambda_{n-1}$ be the principal curvatures of the parallels $N_{r}$ to $N$. Then we can take $B$ to be

$$
B:=2 \max \left\{\left|\lambda_{i}(x)\right|: 1 \leq i \leq n-1, r \in[0, w], x \in N_{r}\right\} .
$$

This is because the linear map $X \mapsto \nabla_{X} \mathbf{n}$ is just the shape operator of $N_{r}$. Using the bound $\left|\left\langle X, \nabla_{X} \mathbf{n}\right\rangle\right| \leq B / 2$ in the calculation above gives the differential inequality

$$
\left|y^{\prime}(t)\right| \leq \frac{B}{2}\left(y(t)^{2}-1\right) .
$$

Using $y(t)=\cosh \left(\rho_{t}\right)$ this can be rewritten as

$$
\left|\frac{d}{d t} \cosh \left(\rho_{t}\right)\right| \leq \frac{B}{2} \sinh ^{2}\left(\rho_{t}\right) .
$$


Lemma 4.7. Let $(M, g)$ be a compact space-like slab. Assume that for some $w>0$ and for each $v \in H(N)$ the inequality $\ell(v) \leq w /|\langle v, \eta(x)\rangle|$ holds. Let $v \in H(N)$, $\gamma(t):=\exp (t v), \rho_{t}=\rho\left(\gamma^{\prime}(t)\right)$, and $y(t)=\cosh \left(\rho_{t}\right)$. Let $y_{0}=y(0)=|\langle v, \eta\rangle|$. Then there is a constant $A \leq(2+B w) / 2$ (where $B$ is the constant in (9)) so that

$$
\frac{1}{A} y_{0} \leq y \leq A y_{0}
$$

Proof. The function $y$ satisfies the differential inequality (9) thus it will be bounded below by the solution to $\bar{y}^{\prime}=-\left(\bar{y}^{2}-1\right) B / 2$ with initial condition $\bar{y}(0)=y_{0}$. The solution is

$$
\bar{y}(t)=\frac{y_{0}+1+\left(y_{0}-1\right) e^{-B t}}{y_{0}+1-\left(y_{0}-1\right) e^{-B t}} .
$$

In this we have $t \leq \ell(v) \leq w /|\langle v, \eta(x)\rangle|=w / y_{0}$. Therefore $e^{-B t} \geq e^{-B w / y_{0}} \geq$ $1-B w / y_{0}$. Using this in the formula for $\bar{y}$ gives

$$
\bar{y} \geq \frac{y_{0}+1+\left(y_{0}-1\right)\left(1-\frac{B w}{y_{0}}\right)}{y_{0}+1-\left(y_{0}-1\right)\left(1-\frac{B w}{y_{0}}\right)} \geq \frac{2 y_{0}}{2+B w}
$$

which is the desired lower bound. To prove the upper bound let $t_{0} \in[0, \ell(v)]$ and define $z(t):=y\left(t_{0}-t\right)$ on the interval $\left[0, t_{0}\right]$. Using the differential inequality (9) for $y$ it is easy to see that $z$ also satisfies $\left|z^{\prime}\right| \leq\left(z^{2}-1\right) B / 2$. Thus the analysis we have just done applies to give

$$
y_{0}=z\left(t_{0}\right) \geq \frac{2}{2+B w} z(0)=\frac{2}{2+B w} y\left(t_{0}\right) .
$$

As $t_{0}$ was any element of the interval $[0, \ell(v)]$ this gives the required upper bound.

Let $n$ be the dimension of $M$. For $a>0$ define functions $h_{a}$ and $f_{a}$ on $[0, \infty)$ by

$$
h_{a}(z):= \begin{cases}1, & 1 \leq z \leq a \\ \frac{a^{n}}{z^{n}}, & a \leq z\end{cases}
$$

and

$$
f_{a}=N_{a} h_{a}
$$

where $N_{a}$ is the constant so that

$$
N_{a} \operatorname{Vol}\left(S^{n-2}\right) \int_{0}^{\infty} h_{a}(\cosh (r)) \sinh ^{n-2}(r) d r=1
$$


To estimate this constant we do the change of variable $x=\cosh (r), d r=d x / \sqrt{x^{2}-1}$ so that for large $a$

$$
\begin{aligned}
\frac{1}{N_{a} \operatorname{Vol}\left(S^{n-2}\right)} & :=\int_{0}^{\infty} h_{a}(\cosh (r)) \sinh ^{n-2}(r) d r \\
& =\int_{1}^{\infty} h_{a}(x)\left(x^{2}-1\right)^{\frac{n-3}{2}} d x \\
& \geq \int_{1}^{a}\left(x^{2}-1\right)^{\frac{n-3}{2}} d x \\
& \geq \begin{cases}\log a, & n=2, \\
\frac{(a-1)^{n-2}}{n-2}, & n \geq 3 .\end{cases}
\end{aligned}
$$

So for large $a$, we have

$$
N_{a} \leq \begin{cases}\frac{1}{\log a}, & n=2, \\ \frac{C(n)}{a^{n-2}}, & n \geq 3 .\end{cases}
$$

Let $\rho: H(M) \rightarrow[0, \infty)$ be as above (that is $\rho(v)$ is the hyperbolic angle that $v$ makes with the vector field $\mathbf{n})$ and we use the function $f(u):=f_{a}(\cosh (\rho(u)))$ in Santaló's formula. Note that if $y \in M$ and $S^{n-2}$ is the unit sphere in $\mathbf{n}(y)^{\perp}$ then vectors $v$ in $H_{y}$ are parameterized by $u=\cosh (\rho(u)) \mathbf{n}(y)+\sinh (\rho(u)) \theta(u)$ where $\theta(u) \in S^{n-2}$. The volume measure on $H_{y}$ is then $d u=\sinh (\rho)^{n-2} d \rho d \theta$, where $d \theta$ is the standard measure on $S^{n-2}$. Thus the normalizing constant in the definition of $f_{a}$ is chosen so that $\int_{H_{y}} f_{a}(\cosh (\rho(v))) d v=1$. Therefore

$$
\int_{M} \int_{H_{y}} f_{a}(\cosh (\rho(u))) d u d x=\operatorname{Vol}(M) .
$$

Let $x \in N, S_{x}^{n-2}=$ unit sphere in $\mathbf{n}(x)^{\perp}$, let $v \in H(N)$ and define $r(v) \in[0, \infty)$ and $\theta(v) \in S_{x}^{n-2}$ by

$$
v=\cosh (r(v)) \mathbf{n}(x)+\sinh (r(v)) \theta(v) .
$$

Let $\rho_{t}(r, \theta):=\rho\left(r, \zeta^{t} v\right)$ with $v$ given by (14) and $\rho, \zeta^{t}$ as above. Then

$$
\begin{aligned}
& \int_{N} \int_{H_{x}} \int_{0}^{\ell(v)}\left(f_{a}\left(\cosh \left(\rho_{t}(r, \theta)\right)-f_{a}(\cosh (\rho(v)))\right) d t|\langle v, \eta(x)\rangle| d v d x\right. \\
&=\int_{N} \int_{S_{x}^{n-2}} I_{a}(x, \theta) d \theta d x
\end{aligned}
$$

where

$$
I_{a}(x, \theta):=\int_{0}^{\infty} \int_{0}^{\ell(v(r, \theta))}\left(f_{a}\left(\cosh \left(\rho_{t}(r, \theta)\right)\right)-f_{a}(\cosh (r))\right) d t \cosh (r) \sinh ^{n-2}(r) d r .
$$

We will suppress the dependence on $x$ and $\theta$. Note that $\rho_{t}=\rho_{t}(r, \theta)$ satisfies $\rho_{0}=r$ and the differential inequality (10) holds. Thus by Lemma $4.7 y(t)=\cosh \left(\rho_{t}\right)$ satisfies

$$
\frac{1}{A} \cosh (r) \leq \cosh \left(\rho_{t}(r)\right) \leq A \cosh (r)
$$


The function $f_{a}$ is constant on the interval $[0, a]$ thus the last inequality implies

$$
\left|f_{a}\left(\cosh \left(\rho_{t}(r)\right)\right)-f_{a}(\cosh (r))\right|=0, \quad \text { for } \cosh r \leq a / A .
$$

For large $r$ we use that from the definition of $f_{a}$ the inequality $\left|f_{a}^{\prime}(z)\right| \leq N_{a} n a^{n} / z^{n+1}$ holds for all $z>0$. Using this bound on $\left|f_{a}^{\prime}\right|$, and the inequalities (10) and (17) gives

$$
\begin{aligned}
\left|f_{a}\left(\cosh \left(\rho_{t}(r)\right)\right)-f_{a}(\cosh (r))\right| & =\left|\int_{0}^{t} \frac{d}{d s} f_{a}\left(\cosh \left(\rho_{s}(r)\right)\right) d s\right| \\
& \leq \int_{0}^{t}\left|f_{a}^{\prime}\left(\cosh \left(\rho_{s}(r)\right)\right) \frac{d}{d s} \cosh \left(\rho_{s}(r)\right)\right| d s \\
& \leq \frac{B}{2} \int_{0}^{t}\left|f_{a}^{\prime}\left(\cosh \left(\rho_{s}(r)\right)\right)\right| \sinh ^{2}\left(\rho_{s}(r)\right) d s \\
& \leq\left(\frac{n B}{2}\right) a^{n} N_{a} \int_{0}^{t} \frac{\sinh ^{2}\left(\rho_{s}(r)\right)}{\cosh ^{n+1}\left(\rho_{s}(r)\right)} d s \\
& \leq\left(\frac{n B}{2}\right) a^{n} N_{a} \int_{0}^{t} \frac{d s}{\cosh ^{n-1}\left(\rho_{s}(r)\right)} \\
& \leq\left(\frac{n A^{n-1} B}{2}\right) a^{n} N_{a} \frac{t}{\cosh ^{n-1}(r)} .
\end{aligned}
$$

We are assuming $\ell(v(r, \theta)) \leq w /|\langle v, \eta\rangle|=w / \cosh (r)$. Then the bounds on

$$
\left|f_{a}\left(\cosh \left(\rho_{t}(r)\right)\right)-f_{a}(\cosh (r))\right|
$$

give

$$
\begin{aligned}
& \int_{0}^{\ell(v(r, \theta))}\left|f_{a}\left(\rho_{t}(r)\right)-f_{a}(r)\right| d t \\
& \quad \leq \begin{cases}0, & \cosh r \leq \frac{a}{A}, \\
\left(\frac{n A^{n-1} B w^{2}}{4}\right) a^{n} N_{a} \frac{1}{\cosh ^{n+1}(r)}, & \frac{a}{A} \leq \cosh r .\end{cases}
\end{aligned}
$$

Using this in the definition of $I_{a}(x, \theta)$ gives

$$
\left|I_{a}(x, \theta)\right| \leq\left(\frac{n A^{n-1} B w^{2}}{4}\right) a^{n} N_{a} \int_{\cosh ^{-1} a / A}^{\infty} \frac{\sinh ^{n-2} r}{\cosh ^{n} r} d r .
$$

For all $n \geq 3$ we have $\sinh ^{n-2} r / \cosh ^{n} r \leq \sinh r / \cosh ^{3} r$. So using the bounds on $N_{a}$ we find for all $n \geq 3$ that

$$
\begin{aligned}
\left|I_{a}(x, \theta)\right| & \leq\left(\frac{n A^{n-1} B w^{2}}{4}\right) a^{n} N_{a} \int_{\cosh ^{-1} a / A}^{\infty} \frac{\sinh r}{\cosh ^{3} r} d r \\
& =\left(\frac{n A^{n-1} B w^{2}}{4}\right) a^{n} N_{a} \frac{1}{2} \frac{A^{2}}{a^{2}} \\
& \leq\left(\frac{n A^{n+1} B w^{2}}{8}\right) C(n) .
\end{aligned}
$$


When $n=2$ for large values of $r$ the inequality $1 / \cosh ^{2} r \leq 2 \sinh r / \cosh ^{3} r$ holds. So using the bound on $N_{a}$ we have for large $a$ that

$$
\begin{aligned}
\left|I_{a}(x, \theta)\right| & \leq\left(\frac{A B w^{2}}{2}\right) a^{2} N_{a} 2 \int_{\cosh ^{-1} a / A}^{\infty} \frac{\sinh r}{\cosh ^{3} r} d r \\
& =\left(A B w^{2}\right) a^{2} N_{a} \frac{A^{2}}{a^{2}} \\
& \leq\left(A^{3} B w^{2}\right) \frac{1}{\log a} .
\end{aligned}
$$

Proof of Theorems 4.2 and 4.3. When $n=2$ defines the function $F_{a}$ on $H(M)$ by $F_{a}(v):=f_{a}(\cosh (\rho(v)))$, then $F_{a}$ is adapted. The estimate (20) implies

$$
\int_{N} \int_{H_{x}} \int_{0}^{\ell(v)}\left(F_{a}\left(\zeta^{t} v\right)-F_{a}(v)\right) d t|\langle v, \eta(x)\rangle| d x \leq C \frac{1}{\log (a)},
$$

for some constant $C<\infty$. Using this in Lemma 4.6 gives

$$
\operatorname{Area}(M)=\lim _{a \rightarrow \infty} \int_{N} \int_{H_{x}} F_{a}(v)|\langle v, \eta(x)\rangle| \ell(v) d v d x
$$

This gives a formula for $\operatorname{Area}(M)$ just in terms of the numbers $\ell(v)$. This implies that if $(M, g)$ and $\left(M^{*}, g^{*}\right)$ are length or lens equivalent then they have the same area which completes the proof of Theorem 4.2.

For the proof of Theorem 4.3 first note if the bound $\ell(v) \leq w /|\langle v, \eta\rangle|$ holds then, with $F_{a}$ as just defined,

$$
\int_{N} \int_{H_{x}} F_{a}(v)|\langle v, \eta(x)\rangle| \ell(v) d v d x \leq w \int_{N} \int_{H_{x}} F_{a}(v) d v d x=w \operatorname{Area}(N) .
$$

The estimate (19) implies for large $a$ that for some constant $C(n)$ only depending on $n$

$$
\int_{N} \int_{H_{x}} \int_{0}^{\ell(v)}\left|F_{a}\left(\zeta^{t} v\right)-F_{a}(v)\right| d t|\langle v, \eta(x)\rangle| d v d x \leq C(n) A^{n+1} B w^{2} \operatorname{Area}(N) .
$$

From Lemma $4.7 A \leq(2+B) / 2$. Using these facts in Lemma 4.6 yields Theorem 4.3.

\section{Boundary Rigidity of Product Manifolds}

Let $(N, h)$ be a complete Riemannian manifold. Recall that the injectivity radius of $(N, h)$ is the supremum of all the numbers $r$ so that the exponential is a diffeomorphism on all balls of radius $r$. The proof of the next result is left to the reader.

Proposition 5.1. Let $(N, h)$ be a complete Riemannian manifold of dimension $(n-1)$. Then the Lorentzian product manifold $(M, g):=\left([0, w] \times N,-d t^{2}+h\right)$ is free of cut points. It is $S G M$ if and only if the injectivity radius of $(N, g)$ is $\geq w$.

Theorem 5.2. Let $(N, h)$ be a compact Riemannian manifold of dimension $(n-1)$ with injectivity radius $\geq w$ and let $(M, g):=\left([0, w] \times N,-d t^{2}+h\right)$. Let $\left(M^{*}, g^{*}\right)$ be a space-like slab boundary isometric to $(M, g)$.

1. If $\operatorname{dim}(M)=2$ then $(M, g)$ and $\left(M^{*}, g^{*}\right)$ are isometric.

2. If $\operatorname{dim}(M) \geq 3$ then $\operatorname{Vol}_{g^{*}}\left(M^{*}\right) \geq \operatorname{Vol}_{g}(M)$ with equality if and only if $(M, g)$ and $\left(M^{*}, g^{*}\right)$ are isometric. 
Proof. We first prove this under the assumption that the past boundary $N^{*}$ of $\left(M^{*}, g^{*}\right)$ is totally geodesic. By the last proposition $(M, g)$ is free of cut points. By Proposition 2.4 this implies $(M, g)$ and $\left(M^{*}, g^{*}\right)$ are lens equivalent. Let $\varphi$ : $\partial M \rightarrow \partial M^{*}$ be the boundary isometry and, to simplify notation, identify $\partial M$ with $\partial M^{*}$ by $\varphi$ and assume that $\partial M=\partial M^{*}$. Let $p \in N$ be a point of the common past boundaries and let $\gamma(t)=\exp (t \eta(p))$ and $\gamma^{*}(t)=\exp \left(t \eta^{*}(p)\right)$ be the normal geodesics to $N$ in $(M, g)$ and $\left(M^{*}, g^{*}\right)$. Let $X_{0}, X_{1} \in T(N)_{p}$ and let $\alpha(s, t)$ be a variation of $\gamma(t)$ through geodesics of $(M, g)$ so that $\alpha(0, t)=\gamma(t)$, $\frac{\partial \alpha}{\partial s}(0,0)=X_{0}$ and $\left(\frac{\nabla}{\partial t} \frac{\partial \alpha}{\partial t}\right)(0,0)=X_{1}$. Let $J(t)=\frac{\partial \alpha}{\partial s}(0, t)$ be the resulting Jacobi field. Let $\alpha^{*}(s, t)$ be a variation of $\gamma^{*}$ through geodesics of $\left(M^{*}, g^{*}\right)$ so that the resulting Jacobi field $J^{*}(t)=\frac{\partial \alpha^{*}}{\partial s}(0, t)$ has the same initial conditions as $J(0)$. Then as $(M, g)$ and $\left(M^{*}, g^{*}\right)$ are lens equivalent we have $J^{*}(w)=J(w)$. For a vector $X(t)$ normal to $\left(\gamma^{*}\right)^{\prime}(t)$, define $R^{*}(t) X:=R^{*}\left(X,\left(\gamma^{*}\right)^{\prime}(t)\right)\left(\gamma^{*}\right)^{\prime}(t)$, where $R^{*}$ is the curvature tensor of $\left(M^{*}, g^{*}\right)$. (Our convention on the sign of curvature is $R(X, Y) Z=\nabla_{X} \nabla_{Y} Z-\nabla_{Y} \nabla_{X} Z-\nabla_{[X, Y]} Z$.) The Jacobi equation along $\gamma^{*}$ is then $X^{\prime \prime}(t)+R^{*}(t) X(t)=0$. Using that $N^{*}$ is totally geodesic in $\left(M^{*}, g^{*}\right)$ if we define a field of linear maps $C_{p}^{*}(t):\left(\gamma^{* \prime}(t)\right)^{\perp} \rightarrow\left(\gamma^{* \prime}(t)\right)^{\perp}$ by

$$
C_{p}^{* \prime \prime}(t)+R^{*}(t) C_{p}^{*}(t)=0, \quad C_{p}^{*}(0)=I, \quad C_{p}^{* \prime}(0)=0,
$$

then the volume of $\left(M^{*}, g^{*}\right)$ is (cf. [11])

$$
\operatorname{Vol}_{g^{*}}\left(M^{*}\right)=\int_{N} \int_{0}^{w} \operatorname{det}\left(C_{p}^{*}(t)\right) d t d p .
$$

As $(M, g)$ is a product manifold and the geodesic $\gamma(t)=\exp (t \eta(p))$ is normal to $N$ we have, with the same notation we have been using, that $R(t)=$ $R\left(\cdot, \gamma^{\prime}(t)\right) \gamma^{\prime}(t) \equiv 0$. As for Jacobi fields $J$ and $J^{*}$ with the same initial conditions $J(w)=J^{*}(w)$ we have that in the terminology of the appendix (cf. Definition 7.1 and the second of the Remarks 7.3) that $R^{*}(t)$ and $R(t) \equiv 0$ are lens equivalent. But then Theorem 7.2 and (21) imply that $\operatorname{Vol}_{g^{*}}\left(M^{*}\right) \geq w \operatorname{Area}(N)$ with equality if and only if $R^{*}(t) \equiv 0$ along all geodesics normal to $N$. But if $R^{*}(t) \equiv 0$ along all geodesics normal to $N$ it follows that $\left(M^{*}, g^{*}\right)$ is isometric to a product $\left([0, w] \times N,-d t^{2}+h\right)$.

When $\operatorname{dim}(M)=2$, Theorem 4.2 implies

$$
\operatorname{Area}_{g^{*}}\left(M^{*}\right)=\operatorname{Area}_{g}(M)=w \operatorname{Length}(N)
$$

so in this case $\left(M^{*}, g^{*}\right)$ and $(M, g)$ are isometric.

It remains to deal with the general case where $N^{*}$ need not be totally geodesic in $\left(M^{*}, g^{*}\right)$. Let $\varepsilon$ be a small positive number. Let $\left(M^{\varepsilon}, g_{\varepsilon}\right)=\left([-\varepsilon, w] \times N,-d t^{2}+h\right)$. Then $(M, g)=\left([0, w] \times N,-d t^{2}+h\right)$ is a subset of $\left(M_{\varepsilon}, g_{\varepsilon}\right)$ in an obvious way. Let $\left(M_{\varepsilon}^{*}, g_{\varepsilon}^{*}\right)$ be the two space-like slabs $\left([-\varepsilon, 0] \times N,-d t^{2}+h\right)$ and $\left(M^{*}, g^{*}\right)$ glued together along $N \times\{0\}=N\left(N \times\{0\}\right.$ is the future boundary of $\left([-\varepsilon, 0] \times N,-d t^{2}+h\right)$ and $N$ is the past boundary of $M)$. The resulting metric $g_{\varepsilon}^{*}$ is continuous and smooth off the hypersurface $N \times\{0\}=N$. (In the terminology of Croke [5] the metric is almost $C^{2}$.) Using the lens equivalence of $(M, g)$ and $\left(M^{*}, g^{*}\right)$ it is not hard to see that $\left(M_{\varepsilon}, g_{\varepsilon}\right)$ and $\left(M_{\varepsilon}^{*}, g_{\varepsilon}^{*}\right)$ are lens equivalent and it is clear that the past boundary $\{-\varepsilon\} \times N$ is totally geodesic in $\left(M_{\varepsilon}^{*}, g_{\varepsilon}^{*}\right)$. The proof given above adapts without changes to almost $C^{2}$ metrics (cf. the appendix to Croke's paper [5]).

Remark 5.3. If $(N, h)$ is a compact Riemannian manifold then the above argument shows that if $(M, g):=\left([0, w] \times N,-d t^{2}+h\right)$ is lens equivalent to the space-like slab 
$\left(M^{*}, g^{*}\right)$ and $\left(M^{*}, g^{*}\right)$ is free of cut points then all the conclusions of Theorem 5.2 still hold. In the Riemannian setting the argument above shows that a compact Riemannian product $(M, g)=\left([0, w] \times N, d t^{2}+h\right)$ is always lens rigid. (It is not hard to see that products $\left([0, w] \times N, d t^{2}+h\right)$ are never strongly geodesically minimizing (SGM) in the sense of [5, Def. 1.1] and we are not making a statement about the boundary rigidity of compact Riemannian products.)

Corollary 5.4. Let $g$ be a Lorentzian metric on $\mathbb{R}^{n}$ that agrees with the standard metric $g_{0}$ outside a compact set $K \subset \mathbb{R}^{n}[0, w]$ and so that $\left(\mathbb{R}^{n}[0, w], g\right)$ is globally hyperbolic, future one connected, and the time-like geodesics of $\left(\mathbb{R}^{n}, g\right)$ have no conjugate points.

1. If $n=2$ then $\left(\mathbb{R}^{2}, g\right)$ and $\left(\mathbb{R}^{2}, g_{0}\right)$ are isometric.

2. If $n \geq 3$ then $\operatorname{Vol}_{g}(K) \geq \operatorname{Vol}_{g_{0}}(K)$ with equality if and only if $\left(\mathbb{R}^{n}, g\right)$ and $\left(\mathbb{R}^{n}, g_{0}\right)$ are isometric.

Proof. Let $D \subset N=\{0\} \times \mathbb{R}^{n-1}$ be a cube with sides of length $L$. If the opposite sides of $D$ are identified the result is an $(n-1)$ dimensional torus $T^{n-1}$ whose injectivity radius is $L / 2$. Choose $L$ so large that $L / 2>w$ and $K \subset[0, w] \times D$. Then by Proposition 5.1 the manifold $\left([0, w] \times T^{n-1}, g_{0}\right)$ is free of cut points and is SGM. The metric $g$ drops down to a metric on $[0, w] \times T^{n-1}$. By Proposition 2.5 the space-like slabs $\left(\mathbb{R}^{n}[0, w], g\right)$ and $\left(\mathbb{R}^{n}[0, w], g_{0}\right)$ are boundary isometric. This implies that $\left([0, w] \times T^{n-1}, g_{0}\right)$ and $\left([0, w] \times T^{n-1}, g\right)$ are boundary isometric. The result now follows from Theorem 5.2.

\section{Proof of the Main Theorem}

In light of Corollary 5.4 to prove Theorem 1.1 it is enough to show

Proposition 6.1. Let $g$ be a Lorentzian metric on $\mathbb{R}^{2}$ that agrees with the standard metric $-d x^{2}+d y^{2}$ outside a compact set $K$. Then there is a conformal diffeomorphism $\varphi:\left(\mathbb{R}^{2}, g\right) \rightarrow\left(\mathbb{R}^{2}, g_{0}\right)$. Thus $\left(\mathbb{R}^{2}, g\right)$ is globally hyperbolic and future one connected.

Remark 6.2. By [1, Proposition 2.30] any Lorentz metric on $\mathbb{R}^{2}$ is stably causal.

Proof. We can assume that $K \subset\{x>0\}$. Then let $V_{+}$be a non-vanishing null (for the metric $g$ ) vector field that agrees with $\partial / \partial x+\partial / \partial y$ on $\mathbb{R}^{2} \backslash K$ and let $V_{-}$be a non-vanishing null vector field vector that agrees with $\partial / \partial x-\partial / \partial y$ on $\mathbb{R}^{2} \backslash K$. These vector fields are bounded with respect to the standard Euclidean structure $d x^{2}+d y^{2}$ on $\mathbb{R}^{2}$ and thus they are complete in the sense that any integral curve of $V_{+}$or $V_{-}$will be defined on all of $(-\infty, \infty)$. Since $\mathbb{R}^{2}$ is simply connected, $[15, \S 3.4$ Lemma 11] shows that null curves intersect at most once. Thus it follows from [1, Proposition 2.28] that the maximal integral curves of $V_{+}$and $V_{-}$intersect exactly once.

Define functions $u, v: \mathbb{R}^{2} \rightarrow \mathbb{R}$ by defined on $\mathbb{R}^{2}$ by the differential equations

$$
\begin{cases}u(0, y)=y, & \text { and } d u\left(V_{+}\right)=0 \\ v(0, y)=y, & \text { and } d v\left(V_{-}\right)=0 .\end{cases}
$$

As $K \subset\{x>0\}$ this implies that on the set $\{x<0\} u(x, y)=-x+y, v(x, y)=$ $x+y$. Define the map $\varphi$ by

$$
\varphi(x, y):=\left(\frac{-u(x, y)+v(x, y)}{2}, \frac{u(x, y)+v(x, y)}{2}\right) .
$$


This map sends null curves to null curves so it is conformal. The $u=$ Const. curves are the integral curves of $V_{+}$and the $v=$ Const. curves are the integral curves of $V_{-}$. As these are all null curves and pairs of null curves meet in at most one point it follows that $\varphi$ is injective.

Let $(a, b) \in \mathbb{R}^{2}$. Let $c_{+}$be the integral curve of $V_{+}$through the point $(0,(-a+b) / 2)$ and $c_{-}$the integral curve of $V_{-}$through $(0,(a+b) / 2)$. Let $\left(x_{0}, y_{0}\right)$ be the unique point where these two curves meet. Then from the definitions $\varphi\left(x_{0}, y_{0}\right)=$ $(a, b)$. Thus $\varphi$ is surjective and this completes the proof that $\varphi:\left(\mathbb{R}^{2}, g\right) \rightarrow\left(\mathbb{R}^{2}, g_{0}\right)$ is a conformal diffeomorphism.

It is clear that a conformal change of metric preserves both global hyperbolicity and future one connectedness. Thus $\left(\mathbb{R}^{2}, g\right)$ is globally hyperbolic and future one connected.

Remark 6.3. We now give an example to show that in dimensions greater than two a Lorentzian metric on $\mathbb{R}^{n}$ agreeing with the standard metric outside a compact set need not be globally hyperbolic, or even causal. For simplicity we work in $\mathbb{R}^{3}$, but the construction can easily be modified to work in higher dimensions. Let $x, y, z$ be the standard coordinates on $\mathbb{R}^{3}$ and let $V_{1}:=\frac{\partial}{\partial x}$ and $V_{2}:=y \frac{\partial}{\partial z}-z \frac{\partial}{\partial y}$. Let $c$ be the circle defined by $x=0, y^{2}+z^{2}=3^{2}$. This is an integral curve for the vector field $V_{2}$. Let $U_{1}$ be the set of points which are at a distance $>1$ from $c$ and $U_{2}$ the set of points at a distance $<2$ from $c$. Then $\left\{U_{1}, U_{2}\right\}$ is an open cover of $\mathbb{R}^{3}$ and the vector fields $V_{1}, V_{2}$ are pointwise linearly independent on the set $U_{1} \cap U_{2}$. Let $\varphi_{1}, \varphi_{2}$ be a partition of unity subordinate to this open cover and set $V:=\varphi_{1} V_{1}+\varphi_{2} V_{2}$. Then the vector field $V$ is non-vanishing, agrees with $V_{2}$ in a neighborhood of $c$ and $V=\frac{\partial}{\partial x}$ outside the ball of radius 5 centered at the origin. Thus $V$ is a vector field that agrees with $\frac{\partial}{\partial x}$ outside a compact set and which has a closed orbit. Because $\mathbb{R}^{3}$ is contractible there are globally defined vector fields $e_{1}, e_{2}, e_{3}$ so that $e_{1} \equiv V$, and outside the ball of radius 5 there holds $e_{1}=\frac{\partial}{\partial x}, e_{2}=\frac{\partial}{\partial y}$ and $e_{3}=\frac{\partial}{\partial z}$. Let $\sigma^{1}, \sigma^{2}$, $\sigma^{3}$ be the dual one forms and let $g=-\left(\sigma^{1}\right)^{2}+\left(\sigma^{2}\right)^{2}+\left(\sigma^{3}\right)^{2}$. Then $g$ is a Lorentzian metric on $\mathbb{R}^{3}$ that agrees with the standard metric $g_{0}=-d x^{2}+d y^{2}+d z^{2}$ outside the ball of radius 5 . As $\mathbb{R}^{3}$ is simply connected the space $\left(\mathbb{R}^{3}, g\right)$ is time orientable and we assume that it has the time orientation that agrees with the standard one outside the ball of radius 5. But then the curve $c$ is a closed time-like curve for the metric $g$ and thus $\left(\mathbb{R}^{3}, g\right)$ is not causal and therefore not globally hyperbolic.

\section{Appendix: Some Results on Ordinary Differential Equations AND SANTALÓ's Formula}

7.1. Lens Equivalent Systems of Differential Equations. We give a precise statement of the results we are using about systems of ordinary differential equations. For the proofs we refer the reader to $[5, \S 6]$. Let $t \mapsto R(t)$ be a continuous map from $[0, w]$ to the space $\mathcal{S}$ of symmetric $m \times m$ matrices. Given such an $R(t)$ we define two matrix valued functions on the interval $[0, w]$ by the initial value problems

$$
\begin{aligned}
& C^{\prime \prime}(t)+R(t) C(t)=0, \quad C(0)=I, \quad C^{\prime}(0)=0, \\
& S^{\prime \prime}(t)+R(t) S(t)=0, \quad S(0)=0, \quad S^{\prime}(0)=I .
\end{aligned}
$$

Definition 7.1. For $i=1,2$ let $t \mapsto R_{i}(t)$ be continuous maps from $[0, w]$ to the space of symmetric matrices. Then $R_{1}(t)$ and $R_{2}(t)$ are lens equivalent on 
$[0, w]$ iff (with the obvious variant of the notation above) both $C_{1}(t)$ and $C_{2}(t)$ are non-singular, all $t \in[0, w]$ and

$$
C_{1}(w)=C_{2}(w), \quad S_{1}(w)=S_{2}(w) .
$$

Theorem 7.2. If $R(t)$ is lens equivalent to $R_{2}(t) \equiv 0$ on $[0, w]$ then

$$
w \leq \int_{0}^{w} \operatorname{det} C(t) d t
$$

and equality holds iff $R(t) \equiv 0$.

Remarks 7.3. 1. In the proof of Proposition 5.2 this is used in the more general case of systems of ordinary differential equations coming from Jacobi equations along geodesics of almost $C^{2}$ metrics. The theorem still holds in this case. We refer the reader to the appendix of Croke's paper [5] for the proof. In the proof of Proposition 5.2 if the past boundary $N^{*}$ of $\left(M^{*}, g^{*}\right)$ is totally geodesic, then the almost $C^{2}$ version of the theorem is not needed. We note that in proving Corollary 5.4 and thus Theorem 1.1 that the manifold $\left(M^{*}, g^{*}\right)$ used in the proof has totally geodesic boundary and we can avoid using the more general version.

2. It follows from elementary linear algebra that $R_{1}(t)$ and $R_{2}(t)$ are lens equivalent on $[0, w]$ iff $\operatorname{det}\left(C_{i}(t)\right) \neq 0$ for $t \in[0, w]$ and for any vectors $v_{0}, v_{1} \in \mathbb{R}^{m}$ if, for $i=1,2, J_{i}(t)$ is the solution of $J_{i}^{\prime \prime}(t)+R_{i}(t) J_{i}(t)=0, J_{i}(0)=v_{0}$ and $J_{i}^{\prime}(0)=v_{1}$, then $J_{1}(w)=J_{2}(w)$. It is this form of lens equivalence that arises naturally in the geometric problems we are considering.

7.2. Santaló's formula. In this section we give a short proof of Santaló's formula in the Lorentzian case which only uses the fact that the geodesic flow is volume preserving and avoids the formalism of integral geometry used in [14, §19.5] or [13]. Let $(M, g)$ be a connected time oriented Lorentzian manifold of dimension $n$ and for each $x \in M$ let $H_{x}$ be the set of all future pointing timelike unit vectors. Each $H_{x}$ is Riemannian manifold isometric to the hyperbolic space of dimension $n-1$ and constant curvature -1 . Give $H(M)$ the measure $\mu$ which is locally the product of the standard measure on the base times the standard measure on the fiber. Thus if $f: H(M) \rightarrow \mathbb{R}$ is a continuous function with compact support

$$
\int_{H(M)} f(v) d \mu(v):=\int_{M} \int_{H_{x}} f(v) d v d x
$$

where $d x$ is the standard volume measure on $M$ and $d v$ is the standard area measure on $H_{x}$.

Let $\zeta^{t}$ be the geodesic flow on $H(M)$. That is if $u \in H(M)$ and $\gamma$ is the geodesic in $M$ with $\gamma^{\prime}(0)=u$, then $\zeta^{t} u=\gamma^{\prime}(t)$. Let $G$ be the vector field on $H(M)$ that generates this flow, that is

$$
G(u)=\left.\frac{d}{d t} \zeta^{t} u\right|_{t=0}
$$

The most basic fact about the geodesic flow is

Theorem 7.4 (Liouville's Theorem). The geodesic flow $\zeta^{t}$ preserves the volume $d \mu$. Thus $\mathcal{L}_{G} d \mu=0$ (where $\mathcal{L}_{G}$ is the Lie derivative with respect to $G$ ).

For a proof of Liouville's theorem that can easily be adapted to cover the present case see [2, Prop. 1.46, p.31]. The next result is just a formula in the differential calculus on manifolds, but it can be viewed as an abstract version of Santaló's formula. The proof is left to the reader. 
Proposition 7.5. Let $M$ be an $m$-dimensional oriented manifold and $\Omega$ an $m$ form on $M$. Let $X$ be a vector field on $M$ so that the Lie derivative $\mathcal{L}_{X} \Omega=0$ and let $\xi^{t}$ be the flow of $X$. Let $S \subset M$ be an oriented hypersurface and define a map $F: S \times \mathbb{R} \rightarrow M$ by $F(x, t):=\xi^{t}(x)$. Then

$$
F^{*} \Omega=\iota_{X} \Omega \wedge d t
$$

where $\iota_{X} \Omega$ is the $m-1$ form obtained by contracting $\Omega$ with $X$.

We now give a more useful formula for the geodesic vector field $G$. Let $\pi$ : $H(M) \rightarrow M$ be the natural projection. For a curve $\gamma:[a, b] \rightarrow H(M)$ let $c=\pi \circ \gamma$. Then $\gamma$ is horizontal iff $\nabla_{c^{\prime}(t)} \gamma \equiv 0$, that is iff $\gamma$ is parallel along $c$. A tangent to $H(M)$ is horizontal iff it is tangent to a horizontal curve. For any vector $Y$ tangent to $M$ there is a unique vector horizontal vector $Y^{\mathcal{H}}$ so that $\pi_{*} Y^{\mathcal{H}}=Y$. The vector $Y^{\mathcal{H}}$ is called the horizontal lift of $Y$. By definition a curve $c$ is a geodesic iff $\nabla_{c^{\prime}(t)} c^{\prime}(t)=0$ and thus it follows that the geodesic vector field is given by

$$
G(u)=u^{\mathcal{H}} .
$$

Lemma 7.6. Let $N$ be an oriented space-like hypersurface in $(M, g)$ with future pointing unit normal field $\eta$. Then $H(N)$ has the measure $d v d x$ which is the product of the measure $d v$ on the fibers and the measure $d x$ on $N$. Then

$$
\left.\iota_{G} d \mu\right|_{T(H(M))}=|\langle v, \eta(x)\rangle| d v d x .
$$

Proof. A straightforward calculation using the form of $G$ given above.

This lemma together with Proposition 7.5 implies that if $H(N) \times \mathbb{R}$ is given the measure $|\langle u, \eta(x)\rangle| d u d x d t$ then the map $(u, t) \mapsto \exp (t u)$ is measure preserving. The form of Santaló's formula given in Theorem 4.5 now follows easily.

\section{REFERENCES}

[1] J. K. Beem and P. E. Ehrlich, Global Lorentzian Geometry, volume 67 of Pure and Applied Mathematics, Marcel Dekker, New York, 1981. MR 82i:53051

[2] A. L. Besse, Manifolds all of whose Geodesics are Closed, volume 93 of Ergebnisse der Mathematik und ihrer Grenzgebiete, Springer-Verlag, New York, 1978. MR 80c:53044

[3] D. Burago and S. Ivanov, Riemannian tori without conjugate points, Geometric and Functional Analysis, 4(3):259-269, 1994. MR 95h:53049

[4] C. Croke, Rigidity for surfaces of nonpositive curvature, Comment. Math. Helv., 65:150-169, 1990. MR 91d:53056

[5] C. Croke, Rigidity and the distance between boundary points, Jour. Diff. Geo., 33:445-464, 1991. MR 92a:53053

[6] L. Green and R. Gulliver, Planes without conjugate points, Jour. Diff. Geo., 22:43-47, 1985. MR 87g:53066

[7] M. Gromov, Filling Riemannian manifolds, Jour. Diff. Geo., 18:1-147, 1983. MR 85h:53029

[8] F. F. Guimarães, The integral of the scalar curvature of complete manifolds without conjugate points, Jour. Diff. Geo, 36:651-662, 1992. MR 93j:53055

[9] S. Hawking and G. F. W. Ellis, The large scale structure of space-time, Cambridge University Press, Cambridge, 1973. MR 54:12154

[10] E. Hopf, Closed surfaces without conjugate points, Proc. Nat. Acad. Sci. U.S.A., 34:47-51, 1948. MR 9:378d

[11] H. Karcher, Riemannian comparison constructions. In S.-S. Chern, editor, Global differential geometry, volume 27, pages 170-222. Mathematical Association of America, Washington, D.C., 1989. MR 91b:53046

[12] R. Michel, Sur la rigiditè imposèe par la longeur des géodésiques, Invent. Math., 65:71-83, 1981. MR 83d:58021 
[13] L. A. Santaló, Integral geometry in general spaces. In Proc. Internat. Congr. Math. Cambridge, Mass., 1950, volume 1, American Math. Soc., Providence, R.I., pages 483-489, 1952. MR 13:377h

[14] L. A. Santaló, Integral Geometry and Geometric Probability, volume 1 of Encyclopedia of mathematics and its applications, Addison-Wesley, Reading, Massachusetts, 1976. MR 55:6340

[15] T. Weinstein, An Introduction to Lorentz Surfaces, Preliminary Manuscript, 1994.

Department of Mathematics, Royal Institute of Technology, S-100 44 Stockholm, SWEDEN

E-mail address: larsa@math.kth.se

Department of Mathematics, Royal Institute of Technology, S-100 44 Stockholm, SWEDEN

E-mail address: dahl@math.kth.se

Department of Mathematics, University of South Carolina, Columbia, South CarOLINA 29208

E-mail address: howard@math.sc.edu 